\title{
Uso de gastrectomía más quimioterapia en cáncer gástrico avanzado con factor único no curable: estudio clínico controlado randomizado fase 3
}

\author{
Alexandra Elimelech F. ${ }^{1}$, Natalia Molina E. ${ }^{1}$, Francisca Aguilera C. ${ }^{1}$, \\ Luis Díaz P. ${ }^{2}$, Arnoldo Riquelme P. ${ }^{2}$ y Gonzalo Pizarro B. ${ }^{3}$
}

Gastrectomy plus chemotherapy in advanced gastric cancer with a single non-curable factor: a phase 3 , randomised controlled trial

\section{Pregunta Clínica}

En los pacientes con cáncer gástrico metastásico, el tratamiento con gastrectomía más quimioterapia con intención paliativa ¿Aumenta la sobrevida en comparación a la lograda con quimioterapia exclusiva?

Para responder a la pregunta clínica, se analiza el siguiente artículo de investigación:

Fujitani K, Yang H, Mizusawa J, Kim Y, Terashima M, Han S, Iwasaki Y, Hyung W, Takagane A, Park D, Yoshikawa T, Hahn S, Nakamura K, Park C, Kurokawa Y, Bang Y, Park B, Sasako M, Tsujinaka T. Gastrectomy plus chemotherapy versus chemotherapy alone for advanced gastric cancer with a single noncurable factor (REGATTA): a phase 3, randomised controlled trial. The Lancet Oncology 2016; 17: 309-18.

\section{Contexto}

El cáncer gástrico es un importante problema de salud a nivel global, corresponde a la quinta causa más común de cáncer en el mundo, y la tercera causa de muerte por cáncer ${ }^{1}$, representando el 8,2\% de muertes por cáncer según estadísticas del GLOBOCAN 2018². Países con infección de Helicobacter pylori endémica, como Japón o Corea del Sur, han demostrado tener éxito en disminución en mortalidad mediante estrategias de screening precoz ${ }^{3}$. Otros factores de riesgo asociados al desarrollo de cáncer gástrico son factores genéticos, infección por virus Epstein Barr, tabaquismo, entre otros.

Se considera cáncer gástrico localmente avanzado a aquel con compromiso T2-T4 y/o N (+), sin metástasis a distancia (M1); y cáncer gástrico metastásico aquel con segundas localizaciones ya sea en hígado, peritoneo, linfonodos distales, entre otros; independiente del T y N. La presencia de metástasis en estos pacientes proporciona un mal pronóstico en la sobrevida global, generalmente menor de 6 meses sin tratamiento sistémico ${ }^{4}$. Sin embargo, metanálisis han demostrado beneficio en sobrevida global de la quimioterapia sistémica (QMT) en comparación a cuidados paliativos exclusivos, con un aumento de sobrevida promedio de 4,3 a 11-13 meses 5 .

En pacientes con adenocarcinoma gástrico metastásico HER2 negativos, la QMT basada en fluoropirimidina más oxaliplatino es la terapia estándar de primera línea para el tratamiento con intención paliativa $^{3}$, pues ha demostrado aumentar la sobrevida global y mejorar la calidad de vida ${ }^{6}$. Sin embargo, estudios previos sugerían que la adición de gastrectomía a quimioterapia -incluso en ausencia de síntomas como hemorragia u obstrucción- podría mejorar la sobrevida de estos pacientes ${ }^{7,8}$. Las guías del National Comprehensive Cancer Network (NCCN) y del Japanese Gastric Cancer Association (JGCA) presentaban diferencias en recomendación de tratamiento a pacientes con enfermedad metastásica. La NCCN sostenía que estos pacientes no eran candidatos a cirugía, a no ser que presentaran complicaciones como obstrucción intestinal o hemorragia grave refractaria a tratamiento médico; mientras la JGCA indicaba que pacientes con metástasis sin síntomas mayores, eran candidatos a gastrectomía. Estudios retrospectivos argumentaban a favor de gastrectomía en cáncer gástrico avanzado, sin embargo con mayor morbilidad y mortalidad relacionada al procedimiento?.

Por otra parte, en relación a los efectos adversos de la gastrectomía, la intervención pudiese retardar el uso de quimioterapia por complicaciones quirúrgicas como filtración de anastomosis, infecciones, retardo en cicatrización ${ }^{3}$ o complicaciones enpostoperatorio como náuseas, vómitos, síndrome de dumping, pérdida de peso y dolor ${ }^{10}$.

Sin embargo, un estudio retrospectivo japonés que
'Escuela de Medicina, Pontificia Universidad Católica de Chile. Santiago, Chile. ${ }^{2}$ Departamento de Gastroenterología, Pontificia Universidad Católica de Chile. Santiago, Chile. ${ }^{3}$ Departamento de Hematología Oncología, Pontificia Universidad Católica de Chile. Santiago, Chile.

Recibido: 11 de julio de 2020 Aceptado: 13 de julio de 2020

Correspondencia a: Dr. Arnoldo Riquelme P. Departamento de Gastroenterología, Facultad de Medicina, Pontificia Universidad Católica de Chile, Marcoleta 367. Santiago, Chile. a.riquelme.perez@ gmail.com 
incluyó a 84 pacientes con cáncer gástrico etapa IV $^{6}$ reporta sobrevida mayor en los pacientes sometidos a cirugía en relación al grupo con quimioterapia exclusiva; además la sobrevida de los pacientes resecados R0 fue de 41,3 meses, valor significativamente superior a los pacientes con resección R1 y R2: 21,2 meses $^{6}$.

En este contexto es necesario evaluar si la adición gastrectomía a la terapia estándar actual de quimioterapia confiere beneficios en la sobrevida en pacientes con cáncer gástrico metastásico.

\section{Métodos}

\section{Características generales}

- Pacientes: Se reclutaron 175 pacientes entre 20 y 75 años de Japón, Corea del Sur y Singapur con adenocarcinoma gástrico T1-3 probado histológicamente, ECOG 0-1 y sin diagnóstico de otro cáncer en los últimos 5 años ni uso de QMT previo. Todos los pacientes contaban con la presencia de un sólo factor no curable confirmado con Tomografía Computada (TC) de abdomen y laparoscopia exploradora o laparotomía. El factor único no curable fue definido como metástasis hepática (2-4 lesiones de diámetro $\leq 5 \mathrm{~cm}$ $\mathrm{y} \geq 1 \mathrm{~cm}$ ); o metástasis peritoneal en diafragma o peritoneo caudal al colon transverso, sin ascitis masiva u obstrucción intestinal; o metástasis de linfonodos para-aórticos bajo arteria mesentérica inferior (diámetro máximo $\geq 1 \mathrm{~cm}$ ). Además, se definió como criterio de inclusión valores de laboratorio de: hemoglobina $\geq 8 \mathrm{~g} / \mathrm{dL}$, plaquetas $\geq 100.000 \mathrm{mcL}, \mathrm{AST}$ o ALT $\leq 100 \mathrm{IU} / \mathrm{L}$, Bilirrubina total $\leq 34,2 \mu \mathrm{mol} / \mathrm{L}$, Creatinina $\leq 106,1 \mu \mathrm{mol} / \mathrm{L} \mathrm{VFG} \geq 60 \mathrm{~mL} / \mathrm{min}$. Todos los pacientes recibieron y firmaron un consentimiento informado.

Se excluyeron aquellos pacientes con metástasis distintas a las mencionadas, coexistencia de otro cáncer activo; cáncer gástrico metastásico HER2 positivo; período de embarazo o lactancia; enfermedades psiquiátricas graves; corticoides sistémicos; tratamiento con flucitosina, fenitoína o warfarina; infección bacteriana o fúngica sistémica activa; angina inestable o infarto al miocardio en los 6 meses pre- vios; hipertensión arterial inestable; diabetes mellitus o enfermedades respiratorias severas dependientes de oxígeno.

- Intervención: Esquema de quimioterapia corresponde a ciclos de 5 semanas con dosis oral de S-1 80 $\mathrm{mg} / \mathrm{m}^{2}$ por día entre días 1 y 21 del ciclo y cisplatino $60 \mathrm{mg} / \mathrm{m}^{2}$ en día 8 (Figura 1). Además, gastrectomía total o parcial dependiendo de localización del tumor con disección de linfonodos D1. Las metástasis en linfonodos perigástricos no fueron disecadas.

- Grupo control: Esquema de quimioterapia corresponde a ciclos de 5 semanas con dosis oral de S-1 80 $\mathrm{mg} / \mathrm{m}^{2}$ por día entre días 1 y 21 del ciclo y cisplatino $60 \mathrm{mg} / \mathrm{m}^{2}$ en día 8 (Figura 1).

- Resultados: Se definió como outcome primario la sobrevida global, entendida como el tiempo transcurrido en meses desde la fecha de randomización hasta la muerte o fecha de último control con paciente sobreviviente. Son outcomes secundarios: sobrevida libre de progresión de enfermedad, seguridad del tratamiento evaluado en efectos adversos y ciclos de quimioterapia por grupo.

- Financiamiento: Ministerio de Salud, Trabajo y Bienestar de Japón y Asociación de Cáncer Gástrico de Corea.

\section{Evaluación de Validez Interna}

- Diseño: Estudio clínico multicéntrico controlado randomizado fase 3 .

- Randomización y ciego: Pacientes elegidos fueron reclutados vía telefónica o fax según la base de datos de Japan Clinical Oncology Group (JCOG), y vía web en la base de datos de I (SNUH) de Corea del Sur y Singapur. Fueron randomizados (1:1) en ramas quimioterapia versus quimioterapia más gastrectomía, mediante el método de biased-coin assignment en grupos balanceados. No hubo ocultamiento de asignación a los pacientes ni investigadores.

- Grupos de estudio: Los pacientes que cumplían con los criterios de elegibilidad fueron randomizados

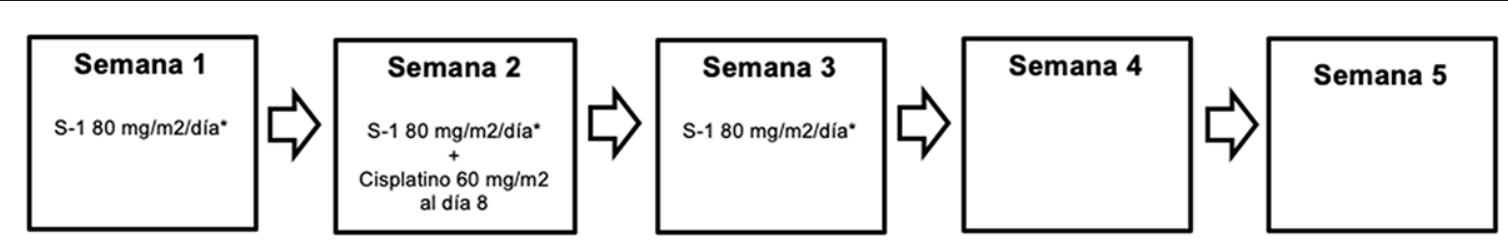

Figura 1. Esquema de quimioterapia recibido por ambos grupos. *Según superficie corporal dosis <1,25 m²: $80 \mathrm{mg} ; 1,25-1,5$ m²: $100 \mathrm{mg}$ y $1,5 \mathrm{~m}^{2}: 120 \mathrm{mg}$. 


\section{Medicina Basada en la evidencia en Gastroenterología}

Tabla 1. Análisis de sobrevida según subgrupos para ambas intervenciones

\begin{tabular}{|lcccccc|}
\hline & QMT (\%) & QMT + G (\%) & HR & p & RR & NNT \\
Sobrevida global & 31,7 & 25,1 & $1,09(\mathrm{O}, 78-1,52)$ & 0,70 & 0,79 & 15,2 \\
$\begin{array}{l}\text { Edad } \\
\quad<60 \text { años }\end{array}$ & 18,0 & 17,5 & $1,56(0,85-2,05)$ & 0,58 & 0,97 & 200 \\
$\quad>60$ años & 16,0 & 18,3 & $0,82(0,52-1,30)$ & & 1,14 & 43,5 \\
País & & & & & & \\
$\quad$ Japón & 17,4 & 12,2 & $1,32(0,85-2,05)$ & 0,18 & 0,70 & 19,2 \\
$\quad$ Corea & 17,5 & 25,0 & $0,85(0,52-1,40)$ & & 1,43 & 133 \\
Localización primario & & & & & & \\
$\quad$ Tercio superior & 12,5 & 6,6 & $2,23(1,14--4,3)$ & 0,027 & 0,53 & 16,9 \\
$\quad$ Tercio medio & 18,4 & 23,3 & $0,95(0,57-1,59)$ & & 1,27 & 20,4 \\
$\quad$ Tercio inferior & 19,0 & 24,1 & $0,63(0,33-1,21)$ & & 1,27 & 196 \\
Etapa clínica & & & & & & \\
$\quad$ N0-1 & 21,3 & 13,3 & $1,79(1,14-2,83)$ & 0,005 & 0,62 & 12,5 \\
$\quad$ N2-3 & 12,8 & 22,7 & $0,70(0,43-1,12)$ & & 1,77 & 10,1 \\
\hline
\end{tabular}

QMT: Quimioterapia, G: Gastrectomfa, HR:Hazard ratio, RR: riesgo relativo, NNT: número necesario a tratar.

en grupos balanceados en base a la institución de origen, etapificación clínica nodal (N0-1 versus N2-3), y factor único no curable; de modo que no hubo diferencias significativas entre ambos grupos de estudio.

- Seguimiento: Se enrolaron un total de 175 pacientes, de los cuales 25 no completaron seguimiento: 12 de quimioterapia exclusiva y 13 de gastrectomía más quimioterapia.

- Escenario: 44 centros de cáncer, hospitales universitarios, hospitales generales y centros médicos en Japón, Corea del Sur y Singapur.

- Tipo de análisis: Por intención de tratar.

- Tiempo de seguimiento: 4 años para reclutamiento de pacientes y 2 años de seguimiento.

- Interrupción precoz: El estudio fue detenido a los dos años debido a análisis provisional por futilidad, ya que no había diferencia significativa de supervivencia global entre los pacientes con quimioterapia exclusiva y los asignados a gastrectomía más quimioterapia. Con ello, el tamaño muestral del análisis primario se redujo a 150 de 175 pacientes, de los 330 originalmente planeados para observar una diferencia de sobrevida global de al menos $10 \%$, limitando el poder estadístico de los resultados.

\section{Resultados}

No hubo diferencias estadísticamente significativas en supervivencia entre el grupo intervenido y control, siendo la sobrevida global a 2 años de $25,1 \%$ para los pacientes sometidos a gastrectomía y $31,7 \%$ para los pacientes tratados con quimioterapia exclusiva. La mediana de sobrevida fue de 16,6 meses para quimioterapia exclusiva versus 14,3 meses para gastrectomía más quimioterapia (HR 1,09, IC $95 \% 0,78-1,52, p=0,70)$. En la Tabla 1 se muestra el análisis de sobrevida por subgrupos diferenciado por edad, país, localización del primario y etapa clínica.

En el análisis de mortalidad por subgrupos las diferencias sólo son relevantes en relación a la localización del tumor, con un $\chi^{2}(2, \mathrm{~N}=175)=9,87$ $\mathrm{y}$ un valor $\mathrm{p}$ significativo $<0,05(\mathrm{p}=0,0072)$. Cabe destacar que el número de ciclos de quimioterapia entregados en ambos grupos, variaron según la ubicación del tumor. En el grupo con tumor ubicado en el tercio superior, donde hubo mayores diferencias, la mediana fue de 3 ciclos para el grupo intervenido y de 6 ciclos para el grupo control, tal y como se muestra en los resultados se muestra en Tabla 2.

Al analizar la mortalidad por subgrupos según edad, país, factor metastásico no curable, etapificación nodal, histología y aspecto macroscópico del tumor, no se encontraron diferencias significativas.

Finalmente, al comparar los efectos adversos en ambos grupos, se calculó el RR individual para cada evento y se sometió a análisis en base al test $\chi^{2}$, resultados que se detallan en la Tabla 3. Al análisis específico, los resultados sólo fueron significativos para mayor tasa de leucopenia y diarrea en pacientes gastrectomizados, con un RR de 1,34 y 1,68 respectivamente. 


\section{Medicina Basada en la evidencia en Gastroenterología}

Tabla 2. Mediana del número de ciclos de quimioterapia en cada grupo y la reducción del grupo experimental con respecto al grupo control, separados por localización del tumor

\begin{tabular}{|cccc|}
\hline Localización tumor & $\begin{array}{c}\text { Mediana ciclos en } \\
\text { quimioterapia exclusiva }\end{array}$ & $\begin{array}{c}\text { Mediana de ciclos en } \\
\text { gastrectomía más quimioterapia }\end{array}$ & $\begin{array}{c}\text { \% reducción ciclos de } \\
\text { quimioterapia }\end{array}$ \\
\hline Tercio superior & $6(4-8)$ & $3(2-5)$ & $50 \%$ \\
Tercio medio & $6(5-8)$ & $5(4-8)$ & $17 \%$ \\
\hline Tercio inferior & $4(2-6)$ & $6(3-8)$ & Aumento $50 \%$ \\
\hline
\end{tabular}

Tabla 3. Eventos adversos hematológicos y quimioterapia exclusiva y quimioterapia más gastrectomía

\begin{tabular}{|c|c|c|c|c|c|}
\hline & QMT $(\mathrm{n}=74)$ & $\mathbf{Q M T}+\mathbf{G}(\mathrm{n}=76)$ & $\mathbf{R R}$ & $\chi^{2}$ & Valor p \\
\hline Leucopenia & $45(61 \%)$ & $62(82 \%)$ & 1,34 & 7,91 & 0,005 \\
\hline Neutropenia & $54(73 \%)$ & $64(84 \%)$ & 1,17 & 2,82 & 0,093 \\
\hline Anemia & $71(96 \%)$ & $75\left(99^{\circ} \%\right)$ & 1,03 & 1,08 & 0,298 \\
\hline Trombocitopenia & $46(62 \%)$ & $53\left(70^{\circ} \%\right)$ & 1,13 & 0,96 & 0,328 \\
\hline Anorexia & $45(61 \%)$ & $54(71 \%)$ & 1,16 & 0,75 & 0,186 \\
\hline Náuseas & $41(55 \%)$ & $41(54 \%)$ & 0,98 & 0,03 & 0,858 \\
\hline Vómitos & $19(26 \%)$ & $22(29 \%)$ & 1,11 & 0,20 & 0,653 \\
\hline Diarrea & $21(28 \%)$ & $36(47 \%)$ & 1,68 & 5,74 & 0,017 \\
\hline Fatiga & $43(58 \%)$ & $44(58 \%)$ & 1 & 0,00 & 0,979 \\
\hline Hiponatremia & $44(59 \%)$ & $41(54 \%)$ & 0,91 & 0,46 & 0,496 \\
\hline
\end{tabular}

QMT: Quimioterapia; G: Gastrectomía; RR Riesgo relativo. $\chi^{2}(1, \mathrm{~N}=150)$.

\section{Discusión}

El presente estudio evalúa la adición de gastrectomía a la quimioterapia convencional con el fin de aumentar la sobrevida global a los pacientes con cáncer gástrico metastásico.

\section{Riesgo de sesgo}

El estudio presenta un adecuado diseño, con un método de randomización que pretendía balancear los tres grupos a estudiar sin intercambio de resultados entre ellos durante el trabajo. El protocolo fue aprobado por el JCOG y la junta de revisión institucional de cada hospital participante. Dos análisis provisionales fueron planeados, tomando en cuenta ajustes para comparaciones repetidas con los métodos Lan y DeMets y O'Brien-Fleming type $\alpha$ spending function. Además los criterios de detención fueron pre-especificados en este estudio.

No obstante, en la presente interpretación de los resultados al evaluar el riesgo de sesgo destaca:

1. Debido al desequilibrio de riesgo existieron dificultades intrínsecas en la acumulación de pacientes por sus criterios de inclusión. Se decidió la detención precoz del estudio por futilidad, mostrando beneficio de la quimioterapia exclusiva sobre el uso de gastrectomía. Sin embargo, al revisar el análisis por subgrupos sólo fue estadísticamente significativo al compararlos según la localización del tumor, siendo que el número de ciclos de quimioterapia fue variable entre ellos, lo que no se descarta como factor que pudiese afectar a sobrevida libre de enfermedad.

2. Dado la morbilidad y riesgo de complicaciones asociado a someterse a una gastrectomía -debidamente informado al paciente-, existe riesgo de sesgo de enmascaramiento tanto para investigadores como participantes ${ }^{12}$.

3. La ubicación del tumor primario no se distribuyó de forma equilibrada entre grupos, pudiendo generar un efecto negativo en la conclusión, pues se observó que hay una interacción significativa entre el efecto del tratamiento y la localización del tumor primario. Este punto debería haberse considerado en la randomización por grupos.

4. No se recogieron datos sobre los parámetros nutricionales de los pacientes.

5. Al tener un grupo intervención con procedimiento quirúrgico y un control sin intervención no es posible que sea un estudio ciego. 


\section{Relevancia de los datos}

Entre $50 \%$ a $60 \%$ de los pacientes con cáncer gástrico debutan con enfermedad metastásica al momento del diagnóstico. Es necesario hacer un balance fino entre los potenciales beneficios en sobrevida al adicionar gastrectomía a la quimioterapia estándar, y los beneficios en la calidad de vida. Previo a la publicación de este artículo existía cierta controversia respecto a este punto, con recomendaciones que sugerían que la gastrectomía adicionada a la quimioterapia podía no solo mejorar los síntomas locales refractarios a tratamiento médico, sino aumentar la sobrevida global. El presente estudio muestra la futilidad de la adición de gastrectomía a la quimioterapia estándar, de hecho existe una mediana de sobrevida global menor a la rama de quimioterapia exclusiva.

Actualmente la gastrectomía en pacientes con cáncer gástrico metastásico está indicada en aquellos pacientes con perforación gástrica y hemorragia digestiva refractaria a manejo médico.

\section{Validez externa}

Los outcomes a evaluar son adecuados en relación a los objetivos de cuidado en enfermedad metastásica, ponderando la sobrevida global en relación a los potenciales efectos adversos de un procedimiento invasivo. Sin embargo, la población objetivo del estudio es población asiática con genética y morbilidades que varían en relación a nuestra población, por lo que se debe evaluar si sus conclusiones son extrapolables.

Por otro lado, dado que el estudio no considera ciego para los pacientes ni investigadores, es importante considerar el efecto Hawthorne, en que los resultados podrían verse afectados por el hecho de saberse parte de un estudio, en relación a los factores situacionales en condiciones normales de la intervención ${ }^{12}$.

No existen otros ensayos controlados aleatorizados que muestren beneficios en sobrevida con la adición de gastrectomía a la quimioterapia exclusiva estándar en pacientes con adenocarcinoma gástrico metastásico.

\section{Conclusión}

La quimioterapia con fluoropirimidina más platino sigue constituyendo el standard of care para el tratamiento con intención paliativa en adenocarcinoma gástrico metastásico HER2 negativo. No existe evidencia que sustente el rol de la cirugía con efectos en la sobrevida global en estos pacientes, además empeora la calidad de vida con mayores efectos adversos. Este estudio Fase III recalca con mayor énfasis la evidencia en contra de gastrectomía ya que fue detenido en análisis por futilidad. De momento el manejo quirúrgico debe reservarse sólo en caso de perforación gástrica y hemorragia digestiva refractaria a manejo médico.

\section{Referencias}

1.- Rawla P, Barsouk A. Epidemiology of gastric cancer: global trends, risk factors and prevention. Prz Gastroenterol. 2019;14:26-38.

2.- World Health Organization. International Agency for Research on Cancer. GLOBOCAN 2018: stomach cancer fact sheet. 2018.

3.- Ajani JA, Lee J, Sano T, Janjigian YY, Fan D, Song S. Gastric adenocarcinoma. Nat Rev Dis Primers. 2017;3:17036.

4.- Van Cutsem E, Sagaert X, Topal B, Haustermans K, Prenen H. Gastric cancer. Lancet. 2016;388:2654- 64.

5.- Wagner AD, Syn NL, Moehler M, Grothe W, Yong WP, Tai BC, et al. Chemotherapy for advanced gastric cancer. Cochrane Database Syst Rev. 2017;8:CD004064.

6.- Yamaguchi K, Yoshida K, Tanahashi T,
Takahashi T, Matsuhashi N, Tanaka Y, et al. The long-term survival of stage IV gastric cancer patients with conversion therapy. Gastric Cancer. 2018;21: 315-23.

7.- Maekawa S, Saku M, Maehara Y, Sadanaga N, Ikejiri K, Anai H, et al. Surgical treatment for advanced gastric cancer. Hepatogastroenterology. 1996;43:178-86.

8.- Fujitani K, Yang HK, Kurokawa Y, Park DJ, Tsujinaka T, Park BJ, et al. Randomized controlled trial comparing gastrectomy plus chemotherapy with chemotherapy alone in advanced gastric cancer with a single non-curable factor: Japan Clinical Oncology Group Study JCOG 0705 and Korea Gastric Cancer Association Study
KGCA01. Jpn J Clin Oncol. 2008;38:504-6.

9.- Mahar AL, Coburn NG, Singh S, Law C, Helyer LK. A systematic review of surgery for non-curative gastric cancer. Gastric Cancer. 2012;15 Suppl1:S125-S137.

10.- Javier M, Loarte A, Pilco P. . Evaluación nutricional en pacientes con gastrectomía total y parcial por Adenocarcinoma gástrico. Rev. gastroenterol. Perú. 2008;28:239-43.

11.- Molina Arias M. Hazard ratio: cuando el riesgo varía a lo largo del tiempo. Rev Pediatr Aten Primaria. 2015; 17 : 185-88.

12.- Higgins JPT, Green S. Cochrane Manual Cochrane de revisiones sistemáticas de intervenciones. Versión 5.1.0 Marzo 2011. The Cochrane Collaboration; 2011. 